\title{
Anyons in geometric models of matter
}

\section{Michael Atiyah $^{a}$ and Matilde Marcolli ${ }^{b}$}

${ }^{a}$ School of Mathematics, The University of Edinburgh, Edinburgh EH9 3FD, Scotland, U.K.

${ }^{b}$ Division of Physics, Mathematics, and Astronomy, California Institute of Technology, 1200 E California Blvd, Pasadena, CA 91125, U.S.A.

E-mail: M.Atiyah@ed.ac.uk, matilde@caltech.edu

ABSTRACT: We show that the "geometric models of matter" approach proposed by the first author can be used to construct models of anyon quasiparticles with fractional quantum numbers, using 4-dimensional edge-cone orbifold geometries with orbifold singularities along embedded 2-dimensional surfaces. The anyon states arise through the braid representation of surface braids wrapped around the orbifold singularities, coming from multisections of the orbifold normal bundle of the embedded surface. We show that the resulting braid representations can give rise to a universal quantum computer.

KEYwords: Anyons, Differential and Algebraic Geometry

ARXIV EPRINT: 1611.04047 


\section{Contents}

1 Introduction 1

2 Orbifolds and geometric models of matter 2

2.1 Edge-cone metrics and orbifolds 2

2.2 Gravitational instantons 3

2.3 Dynamical models 4

2.4 Orbifolds as systems of quasi-particles 5

2.5 Composite systems 6

2.5.1 Connected sums 6

$\begin{array}{ll}\text { 2.5.2 Branched coverings } & 7\end{array}$

$\begin{array}{lll}2.5 .3 & \text { Obstructions } & 7\end{array}$

3 Braid groups, surface braids, and anyons $\quad 8$

3.1 Fundamental groups of surface complements 8

$\begin{array}{llr}3.1 .1 & \text { Orbifold fundamental group } & 9\end{array}$

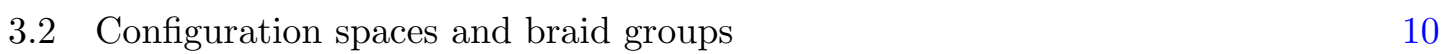

\begin{tabular}{ll}
3.2 .1 & Orbifold braid groups \\
\hline
\end{tabular}

$\begin{array}{lll}3.3 & \text { Surface braids } & 12\end{array}$

$\begin{array}{ll}3.4 & \text { Orbifold normal bundle } \\ 3.5 & 13\end{array}$

$\begin{array}{ll}3.5 & \text { Anyons and vortices } \\ \end{array}$

4 Orbifold edge-cones as a quantum computer 14

$\begin{array}{lll}4.1 & \text { Braided surfaces and universal quantum computers } & 15\end{array}$

$\begin{array}{lll}4.2 & \text { Additional comments and questions } & 17\end{array}$

\section{Introduction}

The main purpose of this paper is to explore new aspects of the geometric approach to models of matter introduced and developed by the first author and collaborators in $[6,10$, 12, 35]. All the mathematical facts we refer to in this paper are known and the reader will be able to find more information about them in the extensive list of references that we provide. The only novelty we introduce is the observation that, within these geometric models of matter, it is possible to describe systems of anyon quasi-particles with fractional quantum numbers, based on orbifold geometries. We show that these geometries allow for the presence of surface braids, wrapped around the 2-dimensional orbifold singularities, and that these surface braids give rise to associated braid representations that determine anyon states. We also show that the anyons that arise from these surface braid configurations can behave like a universal quantum computer. 


\section{Orbifolds and geometric models of matter}

It was shown in [12] that certain classes of 4-dimensional Riemannian manifolds with self-dual Weyl tensor behave in many ways like elementary particles, and can be used to provide geometric models of matter. These manifolds include gravitational instantons like the Taub-NUT manifold [80, 88] or the Atiyah-Hitchin manifold [9], as well as compact manifolds like $\mathbb{C P}^{2}$ or $S^{4}$. These were, respectively, proposed in [12] as models for the proton, the electron, the neutron, and the neutrino. More recent ongoing developments of these geometric models of matter have assigned a different interpretation to some of these manifolds, as we discuss briefly below. These static models of matter were made dynamical in [6], by considering $(4+1)$-dimensional Ricci-flat spacetimes describing evolving TaubNUT geometries. Models of systems of charged particles, based on gravitational instantons of types $A_{k}$ and $D_{k}$ were constructed in [35]. This approach can be viewed, to some extent, as a geometrization of Skyrmion models.

The geometric models of matter originally proposed in [12] have more recently been extended to models of nuclear physics and beta decay, in work of the first author and Nick Manton [10], using algebraic surfaces as geometric models of nuclei, with lepton and baryon numbers related to the topological invariants $c_{2}$ and $c_{1}^{2}$ and to the Enriques-Kodaira classification of compact complex surfaces. In this setting, beta decay is related to blowup operations, and the "valley of stability" is realized by the zero signature region where $c_{1}^{2}=2 c_{2}$. More general models involve non-self-dual cases. In these geometric realizations of atomic level physics, the constituent elementary particles can be obtained when pulling the compact manifold apart by stretching a long neck.

In the present paper, we focus on a different aspect of these geometric models of matter, namely we construct geometric models of systems of quasi-particles, based on 4-dimensional orbifold geometries studied by the first author and LeBrun in [8].

In other recent work of the first author, [2], related to the famous question on the existence of complex structures on the sphere $S^{6}$, odd and even modules for the quaternion group of order eight are considered, where the odd modules are faithful quaternionic representations, with value -1 on the center, while the even ones descend to abelian modules and have value +1 on the center. We expect that these odd and even types will also play a role in the geometric models of matter, where they may be related to topological insulators. We plan to investigate further possible connections to topological insulators and quantum computing aspects of the present work.

\subsection{Edge-cone metrics and orbifolds}

Let $M$ be a smooth compact 4-dimensional manifold and $\Sigma$ a smoothly embedded compact 2 -dimensional surface. According to $[8,65]$, an edge-cone metric on $(M, \Sigma)$ with cone angle $2 \pi \beta$, for some $\beta \in \mathbb{R}_{+}^{*}$, is a smooth Riemannian metric on $M \backslash \Sigma$ that is modelled on a 2-dimensional cone in the directions transversal to $\Sigma$, while it is smooth in the directions parallel to $\Sigma$. More precisely, in a system $\left(\rho, \theta, x^{1}, x^{2}\right)$ of transversal polar coordinates near $\Sigma$, such a metric can be written in the form

$$
g=d \rho^{2}+\beta^{2} \rho^{2}\left(d \theta+u_{j} d x^{j}\right)^{2}+w_{i j} d x^{i} d x^{j}+\rho^{1+\epsilon} h,
$$


where $h$ is a symmetric tensor that has continuous derivatives of all orders with respect to vector fields with vanishing normal component along $\Sigma$ (infinite conormal regularity).

The type of 4-dimensional geometries we are especially interested in here are those that arise as orbifolds. This means a compact 4-dimensional $M$ that admits an atlas of local uniformizing charts $U_{\alpha}$ that are homeomorphic $U_{\alpha} \simeq V_{\alpha} / G_{\alpha}$ to quotients of open sets $V_{\alpha} \subset \mathbb{R}^{4}$ by finite groups $G_{\alpha}$. An orbifold $M$ decomposes $M=M_{\text {sing }} \cup M_{\text {reg }}$ as disjoint union of the set of singular (orbifold) points and the complementary set of regular points. Orbifold points are fixed points of the stabilizer group $G_{\alpha}$ in some chart $U_{\alpha}$. In particular, we consider orbifolds where the set of singular points $M_{\text {sing }}=\Sigma$ is a 2dimensional embedded surface. An orbifold $M$ is a good orbifold if it is a global orbifold quotient, namely there is a smooth 4-manifold $X$ and a finite group $G$ such that $M=X / G$.

Consider an orbifold $(M, \Sigma)$ where $M_{\text {sing }}=\Sigma$ is an embedded surface, and where the orbifold structure near $\Sigma$ can be described in a local uniformizing chart as a quotient $\mathbb{C}^{2} / G_{\nu}$, where $G_{\nu}$ is the cyclic group $G_{\nu}=\mathbb{Z} / \nu \mathbb{Z}$, for some $\nu \geq 2$, with the generator acting by $(w, \zeta) \mapsto\left(w, e^{2 \pi i / \nu} \zeta\right)$. An orbifold edge-cone metric on such an orbifold $(M, \Sigma)$, with cone angle $2 \pi \beta$ and $\beta=1 / \nu$, is an edge-cone metric on the smooth 4-manifold $M \backslash \Sigma$, that is represented in a local chart as a $\mathbb{Z} / \nu \mathbb{Z}$-invariant metric.

A result of [65] shows that if an edge-cone metric with $\beta=1 / \nu$ is an Einstein metric, then it must be an orbifold Einstein metric. Topological obstructions to the existence of Einstein edge-cone metrics were obtained in [8], in terms of index calculations of signature and Euler characteristic. Smooth obstructions to the existence of Einstein edge-cone metrics were then obtained in [65] using Seiberg-Witten theory.

\subsection{Gravitational instantons}

Several examples of 4-dimensional geometries with Einstein edge-cone metrics considered in $[8,65,71]$ also happen to be self-dual or anti-self-dual. We refer to 4-dimensional Riemannian geometries that are both self-dual (or anti-self-dual) and Einstein as gravitational instantons.

A self-dual 4-manifold $M$ has a twistor space $Z=Z(M)$, which is a 3 -dimensional complex manifold that fibers over $M$ with $\mathbb{C P}^{1}$ fibers. This provides an important connection between Riemannian and complex geometry. Moreover, self-dual 4-manifolds behave well under connected sums. Indeed, as shown in [29], the existence of a self-dual metric on a connected sum of two self-dual 4-manifolds, which is close to the original ones outside a neck where the connected sum is performed, can be formulated in terms of twistor geometry. It results from the existence of a deformation of a singular complex 3-manifold obtained by blowing up the twistor spaces $Z_{i}=Z\left(M_{i}\right)$ along a $\mathbb{C P}^{1}$ fiber of the fibration to $M_{i}$ and identifying the exceptional divisors $E_{i}$ of the blowups $\tilde{Z}_{i}$. It is shown in [29] that if the singular space $\tilde{Z}=\tilde{Z}_{1} \cup_{E_{1} \simeq E_{2}} \tilde{Z}_{2}$ admits a smooth Kodaira-Spencer-Kuranishi deformation $Z$, then $Z$ is in fact the twistor space $Z(M)$ of a self-dual structure on the connected sum $M=M_{1} \# M_{2}$.

This connected sum property of self-dual metrics will be useful in the following, to construct systems of quasi-particles. However, for other aspects of the construction we discuss in this paper, it will not be necessary to assume that the 4-dimensional geometries 
we consider are necessarily gravitational instantons, and it is possible to relax this condition for our purposes.

\subsection{Dynamical models}

As mentioned above, the geometric models of matter described in [12] in terms of gravitational instantons, given by 4-dimensional self-dual Riemannian manifolds with an Einstein metric, possibly with edge-cone structure around an embedded surface $\Sigma$, are considered static models. A dynamical version was developed in [6], by embedding the 4-dimensional geometry in a $(4+1)$-dimensional Ricci-flat geometry. The discussion in [6] focuses on the case of the Taub-NUT 4-dimensional gravitational instanton and its embedding in a particular 5-dimensional Ricci-flat geometry that can be viewed as an evolving Taub-NUT geometry, where the parameter of the Taub-NUT metric varies by affine transformations, ensuring the Ricci-flat condition of the 5-dimensional geometry. This 5-dimensional geometry recovers the Sorkin solution of the Kaluza-Klein monopole equations, [87].

More generally, the Campbell-Magaard embedding theorem shows that an arbitrary analytic Riemannian manifold $M$ of dimension $\operatorname{dim} M=n$ can be locally embedded in a Ricci-flat Riemannian manifold of dimension $n+1$, see [70] for a discussion of applications, with particular attention to the embeddings of 4-dimensional (Riemannian) spacetimes in $(4+1)$-dimensional Ricci flat manifolds. The result is extended in [25] to arbitrary signature and to embeddings in Einstein $(n+1)$-dimensional manifolds. All these results only work locally, and the embeddings need not in general extend beyond a small neighborhood of an arbitrary chosen point. Any such embedding can be viewed as a dynamical model for the static geometric model of matter described by the 4-dimensional geometry. In particular, the case of interest to our setting is a 4-dimensional geometry of the form $M \backslash \Sigma$ as above, with an (orbifold) edge-cone metric of cone angle $2 \pi / \nu$, where a Ricci-flat 5 -dimensional embedding extends to a tubular neighborhood $\mathcal{N}(\Sigma) \subset M \backslash \Sigma$ with the edge-cone metric. The analyticity condition is needed for the local embedding. Topological and differentiable obstructions can impose constraints that limit the possibility of extending such embeddings beyond the local existence.

A particular form of the 5-dimensional embedding of an Einstein 4-dimensional manifold $M$ with $R_{i j}=-\lambda g_{i j}$ is given in [25] section III, with the 5-dimensional metric of the form

$$
d s^{2}=f(u) g_{i k} d x^{i} d x^{k}+\epsilon d u^{2}
$$

with $\epsilon= \pm 1$ and with

$$
f(u)=\left(\cosh \left((-\epsilon \Lambda / 6)^{1 / 2} u\right)+(1-2 \lambda / \Lambda) \sinh \left(\left((-\epsilon \Lambda / 6)^{1 / 2} u\right)\right)^{2},\right.
$$

depending on $\epsilon, \Lambda, \lambda$, where $\Lambda$ is the cosmological constant of the Einstein metric on the 5 -dimensional geometry. A similar geometric setting is considered in the context of brane world collisions, [42].

One can consider, in addition to embeddings of the 4-dimensional geometry $M$ into a 5-dimensional product geometry $M \times I$, the possibility of topology changes, namely of embeddings into topologically non-trivial 5 -dimensional cobordisms. Physically acceptable 
conditions on the existence of such topology changes are analyzed in [30], in terms of handlebody decompositions of the cobordism. Possibilities include the case of pair production of Kaluza-Klein monopoles, with the topology change from $S^{3} \times S^{1}$ to $S^{4}$ via a $D^{5} \backslash S^{1} \times D^{4}$, which admits Riemannian as well as causally continuous almost Lorentzian metrics, constructed using a Morse function (see [30]).

\subsection{Orbifolds as systems of quasi-particles}

A main reason for interpreting these types of 4-dimensional orbifolds as geometric models of quasi-particles is the presence of fractional quantum numbers. In the approach of [12] to geometric models of matter, the signature $\tau(M)$ is interpreted as a baryon number, while the electric charge is determined by the self-intersection number of the surface at infinity. The Euler characteristic, on the other hand, does not play a direct role as a quantum number in the geometric models of [12], unlike what typically happens in geometric models of the quantum Hall effect, where it is related to the noncommutative Kubo formula for the transport coefficient $([19,24,74-77])$. In the more recent work of the first author and Nick Manton [10], for models of matter based on algebraic surfaces, baryon and lepton numbers are expressed in terms of both signature and Euler characteristic, with the signature measuring the difference between the number of protons and the number of neutrons. The models considered in [12] dealt with conformally self-dual manifolds. For these, the definitions agree with those of [10].

Let $M$ be a 4-dimensional compact orbifold $M$ with set of orbifold points given by an embedded surface $\Sigma$, endowed with a self-dual (or anti-self-dual) orbifold edge-cone metric with cone angle $2 \pi / \nu$ with $\nu \in \mathbb{N}, \nu \geq 2$. Let $W$ denote the Weyl tensor, with $W^{ \pm}$ the decomposition into self-dual and anti-self-dual part, $E$ the traceless part of the Ricci tensor, and $R$ the scalar curvature. In [8] it is shown that one obtains an orbifold Euler characteristic and an orbifold signature, respectively given by

$$
\begin{aligned}
& \chi_{\mathrm{orb}}(M)=\frac{1}{8 \pi^{2}} \int_{M}\left(|W|^{2}-\frac{1}{2}|E|^{2}+\frac{1}{24} R^{2}\right) d v(g)=\chi(M)-\left(1-\frac{1}{\nu}\right) \chi(\Sigma), \\
& \tau_{\mathrm{orb}}(M)=\frac{1}{12 \pi^{2}} \int_{M}\left(\left|W^{+}\right|^{2}-\left|W_{-}\right|^{2}\right) d v(g)=\tau(M)-\frac{1}{3}\left(1-\frac{1}{\nu^{2}}\right)[\Sigma]^{2}
\end{aligned}
$$

where $[\Sigma]^{2}$ is the self-intersection number. As discussed in $[8,71]$, the orbifold Euler characteristic and signature of (2.3) and (2.4) fit in the framework of the Kawasaki index theorem for orbifolds [56]. Namely, as shown in [71], there is an elliptic complex $\mathcal{K}$, which depends on the orbifold $M$ and the edge-cone metric, such that

$$
\operatorname{Ind}(\mathcal{K})=\operatorname{dim} H^{0}-\operatorname{dim} H^{1}+\operatorname{dim} H^{2}=\frac{1}{2}(15 \chi(M)-29 \tau(M))-4 \chi(\Sigma)+4[\Sigma]^{2} .
$$

The quantities $\chi_{\text {orb }}(M)$ and $\tau_{\text {orb }}(M)$ should be interpreted as fractional quantum numbers for the orbifold $M$, viewed as modeling a system of quasi-particles.

The self-intersection number $[\Sigma]^{2}$ is the same as the Euler number of the normal bundle of $\Sigma$ in $M$. In the complement $M_{\text {reg }}=M \backslash \Sigma$, the surface $\Sigma$ of orbifold points can be viewed, by analogy with the geometric models of matter described in [12], as being the 
surface at infinity that contributes the electric charge to the matter content. In order to take into account the orbifold structure properly, note that the normal bundle $\mathcal{N}(\Sigma)$ of the inclusion of $\Sigma$ in $M$ is an orbifold vector bundle, whose fibers are quotients $\mathbb{R}^{2} / G_{\nu}$ where $G_{\nu}=\mathbb{Z} / \nu \mathbb{Z}$ is the stabilizer of $\Sigma$. Thus, the self-intersection number should be replaced by the orbifold Euler number of the normal bundle $\mathcal{N}(\Sigma)$, that is, $[\Sigma]_{\text {orb }}^{2}=\chi_{\text {orb }}(\mathcal{N}(\Sigma))$. This is the rational valued Satake orbifold Euler characteristic [84], rather than the integer valued orbifold Euler characteristic of good orbifolds $M=X / G$ considered in [13] and [44]. The fractional quantum number $[\Sigma]_{\text {orb }}^{2}=\chi_{\text {orb }}(\mathcal{N}(\Sigma))$ represents the fractional electric charge of the system of quasi-particles. In ordinary matter, quarks exhibit both fractional electric charge and fractional baryon numbers. Fractional baryon numbers occur in quark-gluon plasma, in skyrmion models, and in models of baryogenesis from scalar condensates.

In the geometric models of matter developed in $[6,10,12]$ the interpretation of the signature as baryon number is compatible with viewing baryon number as arising through a chiral symmetry breaking in QCD. The way in which the chiral symmetry breaking is implicitly built into these models is through an index theorem. Indeed, it is well known (see for instance [18] and the survey [17]) that the axial anomaly is a topological density given by the Chern-Pontryagin class and the integrated form of the anomalous axial current is expressed as an index theorem of a Dirac operator. Thus, for instance, the fact that on Taub-NUT gravitational instantons the relevant index is zero implies that chiral symmetry breaking is not present, and this is consistent with the interpretation given in [6] of the Taub-NUT as a geometric model for a particle with zero baryon number. In the setting we consider here the chiral symmetry breaking is still included in the model in the form of an index theorem, but due to the presence of an orbifold structure, the relevant form in which it appears is a Kawasaki index theorem for orbifolds, [56].

\subsection{Composite systems}

Interpreting, as above, the 4-dimensional orbifolds as quasi-particles allows for interesting constructions of composite systems of quasi-particles, arising from natural geometric constructions of 4-manifolds. However, as shown in [8, 65, 71], there are obstructions to the existence of Einstein metrics on these composite systems, so it is not always possible to obtain such systems as gravitational instanton models of matter. Some of the available constructions that give rise to quasi-particle systems are connected sums and branched coverings. We can regard these operations as ways of obtaining composite systems, respectively, by a merging (fusion) and by a branching operation. We describe these more in detail.

\subsubsection{Connected sums}

As we recalled above, it is possible to endow with a self-dual metric a connected sum of two self-dual 4-manifolds [29], under suitable conditions that can be identified in terms of twistor spaces. The argument of [29] based on deformation theory was extended to the case of self-dual 4-dimensional orbifolds in [62, 66, 71]. In particular, an edge-cone metric is unobstructed if $H^{2}=0$ in the complex $\mathcal{K}$ of $(2.5)$. If $\left(M_{1}, \Sigma_{1}\right)$ and $\left(M_{2}, \Sigma_{2}\right)$ are unobstructed self-dual orbifolds, with set of orbifold points $\Sigma_{i}$ and with edge-cone metrics with the same cone angle $2 \pi / \nu$, then the connected sum $\left(M_{1} \# M_{2}, \Sigma_{1} \# \Sigma_{2}\right)$ can also be 
endowed with an unobstructed self-dual orbifold-cone metric with the same cone angle and set of orbifold points $\Sigma_{1} \# \Sigma_{2}$. This operation should be regarded as a way of merging the systems of quasi-particles represented by the orbifolds $\left(M_{1}, \Sigma_{1}\right)$ and $\left(M_{2}, \Sigma_{2}\right)$ into a combined system.

\subsubsection{Branched coverings}

One can view branched coverings as orbifold coverings. Thus, one can obtain 4-dimensional orbifolds $M$ where the set of orbifold points is given by an embedded (not necessarily connected) surface $\Sigma$ by considering smooth 4-manifolds that arise as branched coverings of $M$ with branch locus $\Sigma$. In this type of construction, the branch locus $\Sigma$ is usually embedded in a way that can be highly knotted. Indeed, a branched covering of order $n$ is determined by a representation of the fundamental group $\pi_{1}(M \backslash \Sigma)$ (which carries the information on the amount of knottedness of the embedding) in the symmetric group $S_{n}$. It is known by the result of [48] that any orientable closed PL 4-manifold is a 5-fold simple branched covering of $S^{4}$, branched along an embedded surface $\Sigma$. Moreover, it is known by [16] that every compact symplectic 4 -manifold is a branched cover of $\mathbb{C P}^{2}$ branched along a symplectic curve (immersed with cusps) in $\mathbb{C P}^{2}$. In [4] it was shown that the projective planes $\mathbb{P}^{2}(\mathbb{C}), \mathbb{P}^{2}(\mathbb{H})$ and $\mathbb{P}^{2}(\mathbb{O})$ are branched coverings of $S^{4}, S^{7}$ and $S^{13}$, with branch locus respectively given by $\mathbb{P}^{2}(\mathbb{R}), \mathbb{P}^{2}(\mathbb{C})$ and $\mathbb{P}^{2}(\mathbb{H})$. Passing from an orbifold geometry $(M, \Sigma)$ to an orbifold covering $\left(M^{\prime}, \Sigma^{\prime}\right)$ can be viewed as another way of obtaining composite systems of quasi-particles.

\subsubsection{Obstructions}

There are obstructions to be taken into account when forming composite systems of quasiparticles with the methods described above. The first type of obstruction to be taken into consideration arises in the deformation argument that ensures the existence of selfdual metrics on the connected sum. For instance, there are no unobstructed self-dual orbifold-cone metrics on $S^{4}$ with set of orbifold points an orientable embedded surface $\Sigma$ of genus $g \geq 1$ (Corollary 1.9 of [71]), while such unobstructed metrics exist for $\Sigma=S^{2}$ and $\Sigma=\mathbb{R P}^{2}$, see [8], and for any connected sum of an arbitrary number of $\mathbb{R P}^{2}$, see [71].

If we insist on the requirement that the geometric models of matter should be gravitational instantons, namely both self-dual and Einstein, then there are also obstructions to the existence of Einstein metrics that one needs to take into account. These obstructions can be of topological nature or of differentiable nature. Topological obstructions have been identified in [8]: the inequalities

$$
2 \chi(M) \pm 3 \tau(M) \geq\left(1-\frac{1}{\nu}\right)\left(2 \chi(\Sigma) \pm\left(1+\frac{1}{\nu}\right)[\Sigma]^{2}\right),
$$

have to be satisfied for a 4-dimensional orbifold $(M, \Sigma)$ to admit an Einstein edge-cone metric of cone angle $2 \pi / \nu$. Differentiable obstructions have been identified in [65] using Seiberg-Witten theory: if $M$ admits a symplectic form $\omega$ for which $\Sigma$ is a symplectic submanifold with $\left(c_{1}(M)-(1-1 / \nu)[\Sigma]\right) \cdot[\omega]<0$, then for any $\ell \geq\left(c_{1}(M)-(1-1 / \nu)[\Sigma]\right)^{2} / 3$ the pair $\left(M^{\prime}, \Sigma\right)$ with $M^{\prime}=M \#^{\ell} \overline{\mathbb{C P}^{2}}$ does not admit an Einstein edge-cone metric (Theorem A of $[65])$. 
For the reason described here above, the operation of connected sum that plays the role of fusion rules giving rise to composite systems is not always well defined, in an unobstructed way, within the class of gravitational instantons. Thus, it seems preferable, within the context of obtaining sufficiently well behaved systems of quasi-particle, to relax the assumptions that all the 4-manifolds involved are gravitational instantons, and allow for a larger class of 4-manifolds with edge-cone orbifold geometries.

\section{Braid groups, surface braids, and anyons}

In the previous section, we described geometric models of systems of quasi-particles in terms of 4-dimensional gravitational instantons $M$ with an orbifold structure and an edgecone metric around an embedded surface $\Sigma$ of orbifold points. In this section we show that these models exhibit interesting braiding structures that behave like physical anyons. It is well known that anyons arise only in two-dimensional systems, due to the topology of configuration spaces that allows for interesting braid groups, see [47, 60, 67]. Indeed, anyons and representations of braid groups have been considered in relation to quantum Hall systems, $[49,50,77]$. While anyons do not arise in higher dimensions, we will see that the presence of the 2-dimensional surfaces $\Sigma$ of orbifold points allows for the existence of non-trivial anyon states and interesting braid group representations associated to the 4-dimensional orbifold geometries that describe our quasi-particle systems. We begin by analyzing the different forms of knottedness and braiding that are present in our geometric setting and the role they play in describing properties of the corresponding quasi-particle system.

\subsection{Fundamental groups of surface complements}

An embedded 2-dimensional surface $\Sigma$ in a 4-dimensional manifold $M$ has an associated fundamental group $\pi_{1}(M \backslash \Sigma)$. In the case of a 2-knot, that is, an embedding $\iota: S^{2} \hookrightarrow S^{4}$, the fundamental group $\pi_{1}\left(S^{4} \backslash \iota\left(S^{2}\right)\right)$ plays a role analogous to the knot groups $\pi_{1}\left(S^{3} \backslash K\right)$ that measure the amount of knottedness of embeddings $K$ of $S^{1}$ in $S^{3}$. Explicit presentations for fundamental groups $\pi_{1}\left(S^{4} \backslash \Sigma\right)$, with $\Sigma$ an embedded surface, are obtained in [58], by combining the use of Wirtinger presentations of knot groups in 3-dimensions and van Kampen's theorem.

In the case of 2-dimensional surfaces $\Sigma$ embedded in smooth 4-dimensional manifolds, a phenomenon arises that is not present in the more familiar lower dimensional case, namely the possibility of exotic knottedness: this refers to embedded surfaces that are topologically but not smoothly isotopic, see [34, 57]. Since we are mostly interested here in discussing topological properties, and in particular representations of knot and braid groups arising from embeddings of surfaces in 4-manifolds, we do not need to worry about the possible effect of exotic smoothness and knottedness. However, exotic smoothness can influence other aspects of the geometry, such as conditions on the existence of appropriate metrics.

Both the knot groups $\pi_{1}\left(S^{3} \backslash K\right)$ and the fundamental groups $\pi_{1}\left(S^{4} \backslash \Sigma\right)=\pi_{1}\left(\mathbb{R}^{4} \backslash \Sigma\right)$, with $\Sigma$ an embedded surface, are examples of a larger class of groups, called $C$ groups, see [63]. These are defined by the existence of a presentation of the form $\left\langle x_{1}, \ldots, x_{n} \mid R_{\alpha}\right\rangle$ where the relations $R_{\alpha}$ with $\alpha=\left(\alpha_{i}\right)_{i=1,2,3}$ are all conjugations of the 
form $R_{\alpha}=x_{\alpha_{1}} x_{\alpha_{2}} x_{\alpha_{1}}^{-1} x_{\alpha_{3}}^{-1}$. If $\Sigma$ is a smoothly embedded 2-dimensional orientable compact surface in $S^{4}$, then $\pi_{1}\left(S^{4} \backslash \Sigma\right)$ is a $C$-group whose abelianization is $\pi_{1}\left(S^{4} \backslash \Sigma\right)^{a b}=\mathbb{Z}^{k}$, where $k$ is the number of components of $\Sigma$, see Theorem 1 of [63]. It is shown in [63] that any $C$-group can be realized as $\pi_{1}\left(S^{4} \backslash \Sigma\right)$, with $\Sigma$ an embedded surface, though not all $C$-groups are fundamental groups of complements of 2-knots $S^{2} \hookrightarrow S^{4}$. However, as we pointed out before, the complements $S^{4} \backslash \Sigma$ with $g(\Sigma) \geq 1$ do not carry unobstructed self-dual metrics, by [71], so not all these possibilities will arise from geometric models of quasi-particle systems with good composition properties. In any case, the general result of [63] shows that the class of fundamental groups $\pi_{1}(M \backslash \Sigma)$ can be highly nontrivial.

However, there are also many significant examples where the fundamental group $\pi_{1}(M \backslash \Sigma)$ does not carry enough interesting information. For instance, in the case of an embedding of an algebraic curve $C$ in the plane $\mathbb{C}^{2}$, if the curve is smooth, then the fundamental group $\pi_{1}\left(\mathbb{C}^{2} \backslash C\right)$ is cyclic generated by a loop transverse to the curve. It was shown in $[27,40]$ that even in the case of nodal singularities the fundamental group of the complement is abelian. This fact suggests that, while generally interesting, the groups $\pi_{1}(M \backslash \Sigma)$ are not the correct fundamental groups to consider in our setting, to obtain a physical system with interesting fractional statistics.

For more general singular plane curves, however, the fundamental group of the complement is not necessarily abelian and can be very interesting. A significant example was already described by Zariski in [93] (see also [94]): in the case where $C$ is a sextic with six cusps, if the cusps lie on a conic, the fundamental group of the complement is $\mathbb{Z} / 2 \mathbb{Z} \star \mathbb{Z} / 3 \mathbb{Z}$, while if the six cusps do not lie on a conic the fundamental group is $\mathbb{Z} / 2 \mathbb{Z} \times \mathbb{Z} / 3 \mathbb{Z}$. A cohomological interpretation of the Zariski example is discussed, for instance, in section 4 of [28].

Moreover, for singular curves there is an interesting relation between the fundamental group $\pi_{1}\left(\mathbb{C}^{2} \backslash C\right)$ and braid groups, in the form of braid monodromy. Unlike the case of a smooth or nodal algebraic curve $C$ in $\mathbb{C}^{2}$, for a more general planar curve with arbitrary singularities, the fundamental group $\pi_{1}\left(\mathbb{C}^{2} \backslash C\right)$ has a more complicated structure. For the general case of singular algebraic curves $C$ in $\mathbb{C}^{2}$, a presentation of $\pi_{1}\left(\mathbb{C}^{2} \backslash C\right)$ was constructed in [69], with the property that the 2-dimensional $\mathrm{CW}$ complex associated to the group presentation, with one 0-cell, a 1-cell for each generator and a 2-cell for each relation, is homotopy equivalent to the complement $\mathbb{C}^{2} \backslash C$. The presentation is based on the braid monodromy construction of [78]. A linear projection of $\mathbb{C}^{2}$ onto a line $L=\mathbb{C}$ determines a locally trivial bundle $\mathbb{C}^{2} \backslash C \rightarrow L$ and a homomorphism (the braid monodromy) of $\pi_{1}\left(\mathbb{C}^{2} \backslash C\right)$ to the group of diffeomorphisms of the fiber that fix the intersection with $C$, identified with a braid group, see [69, 78]. We will see below that a similar structure with braid group representations will provide the source of anyons in our models.

\subsubsection{Orbifold fundamental group}

In general if $M$ is a good orbifold, with singular locus $M_{\text {sing }}=\Sigma$ of real codimension two, the orbifold fundamental group $\pi_{1}^{\text {orb }}(M)$ is given (see [89], section 13) by the quotient

$$
\pi_{1}^{\mathrm{orb}}(M)=\pi_{1}\left(M_{\mathrm{reg}}\right) / H
$$


where $M_{\text {reg }}=M \backslash M_{\text {sing }}=M \backslash \Sigma$ is the set of regular points of the orbifold and $H$ is the normal subgroup generated by the classes $\gamma_{j}^{\nu_{j}}$ in $\pi_{1}\left(M_{\text {reg }}\right)$, where the $\gamma_{j}$ are loops encircling the connected components $M_{\text {sing, } j}=\Sigma_{j}$ of $M_{\text {sing }}=\Sigma$, and $\nu_{j} \in \mathbb{N}$ is the order of the stabilizer $G_{j}$ of the component $M_{s i n g, j}$. Thus, in the kind of geometry that we consider here, the fundamental group $\pi_{1}(M \backslash \Sigma)$ considered above, should be replaced by the orbifold fundamental group $\pi_{1}^{\text {orb }}(M)=\pi_{1}(M \backslash \Sigma) / H$.

One can view the sphere $S^{4}$ as a quotient of $\mathbb{C P}^{2}$ by $\mathbb{Z} / 2 \mathbb{Z}$ with branch locus $\mathbb{R} \mathbb{P}^{2}$ (see [4] for similar descriptions of $S^{7}$ and $S^{13}$ ). On the complement $M \backslash \Sigma=S^{4} \backslash \mathbb{R P}^{2}$ consider the Hitchin family of self-dual Einstein orbifold edge-cone metrics with cone angle $2 \pi /(k-2)$, constructed in [45]. The fundamental group is $\pi_{1}\left(S^{4} \backslash \mathbb{R} \mathbb{P}^{2}\right)=\mathbb{Z} / 2 \mathbb{Z}$. When $k$ is even $\pi_{1}^{\text {orb }}(M)=\mathbb{Z} / 2 \mathbb{Z}$, while it is trivlal when $k$ is odd. The same holds for composite systems obtained by connected sums, with $M_{\text {reg }}=S^{4} \backslash \#^{\ell} \mathbb{R P}^{2}$, with $\pi_{1}\left(M_{\text {reg }}\right)=\mathbb{Z} / 2 \mathbb{Z}$. As another example, consider the Atiyah-LeBrun edge-cone metrics defined in [8] p.21, on $M_{\text {reg }}=S^{4} \backslash S^{2}$, with the standard unknotted embedding of $S^{2}$, with cone angle $2 \pi / \nu$. The associated orbifold fundamental group is $\pi_{1}^{\text {orb }}(M)=\mathbb{Z} / \nu \mathbb{Z}$.

It is clear from these examples that, in such cases of interesting geometric models of matter with orbifold structure, the orbifold fundamental group is too simple to give rise to any interesting braiding that can be interpreted in terms of anyon states. We will review briefly the relation between braid groups, fractional statistics and anyons, and then we will revisit the geometry of the embedded surfaces $\Sigma=M_{\text {sing }}$ in order to identify the correct source of interesting braiding.

\subsection{Configuration spaces and braid groups}

Let $X$ be a smooth manifold. Consider the space $F_{n}(X)=X^{n} \backslash \Delta$, that is, the complement of the diagonals in $X^{n}$,

$$
F_{n}(X)=\left\{\left(x_{1}, \ldots, x_{n}\right) \in X^{n} \mid x_{i} \neq x_{j}, \forall i \neq j, i, j=1, \ldots, n\right\}
$$

The symmetric group $S_{n}$ acts freely on $F_{n}(X)$. The configuration spaces of $X$ are the quotients

$$
\operatorname{Conf}_{n}(X):=F_{n}(X) / S_{n} .
$$

Configuration spaces play an important role in physical models, where they describe distinct point particles and provide a geometric setting for quantum mechanical spin-statistics results, see [1, 5, 14, 15]. The Pauli sign for fermions was interpreted in [20], using Schwinger representations of spin, as a topological phase arising from noncontractible loops in a nonorientable configuration space. In the context of geometric models of matter, the $n=$ 2 configuration space plays an important role in the construction of [3]. Configuration spaces also provide the background for the formulation of the multi-Taub-NUT geometries considered in [6], which can be regarded as a geometric approach towards some of the ideas in Feynman's thesis [23].

The braid groups of $X$ are the fundamental groups

$$
B_{n}(X):=\pi_{1}\left(\operatorname{Conf}_{n}(X)\right) .
$$


The quotient description (3.2) implies that the fundamental groups are related by a sequence

$$
1 \rightarrow \pi_{1}\left(F_{n}(X)\right) \rightarrow \pi_{1}\left(\operatorname{Conf}_{n}(X)\right) \rightarrow S_{n} \rightarrow 1,
$$

while the higher homotopy groups satisfy $\pi_{i}\left(F_{n}(X)\right)=\pi_{i}\left(\operatorname{Conf}_{n}(X)\right)$ for $i \geq 2$.

If $X$ is a manifold of dimension $m=\operatorname{dim} X>2$, then it is known (see for instance [53] and [47]) that $\pi_{1}\left(F_{n}(X)\right)=\pi_{1}(X)^{n}$ and that the braid group is a wreath product

$$
B_{n}(X)=\pi_{1}(X) \prec S_{n}=\pi_{1}(X)^{n} \rtimes S_{n},
$$

while the higher homotopy groups satisfy

$$
\pi_{i}\left(\operatorname{Conf}_{n}(X)\right)=\pi_{1}(X)^{n}, \quad \text { for } 2 \leq i \leq m-2,
$$

see Theorem 1.2 of [53].

In particular, we are interested in the case where $X=M \backslash \Sigma$ where $M$ is a smooth compact 4-manifold and $\Sigma$ is a smoothly embedded compact 2-dimensional surface (not necessarily connected). In this case we have

$$
B_{n}(M \backslash \Sigma)=\pi_{1}(M \backslash \Sigma) \imath S_{n},
$$

A presentation of $\pi_{1}(M \backslash \Sigma)$ can then be used to obtain an explicit presentation for the braid groups $B_{n}(M \backslash \Sigma)$. For the second homotopy group we simply have

$$
\pi_{2}\left(\operatorname{Conf}_{n}(M \backslash \Sigma)\right)=\pi_{2}(M \backslash \Sigma)^{n} .
$$

For example, if $\iota: S^{2} \hookrightarrow S^{4}$ is a smoothly embedded 2-knot in $S^{4}$, such that $\pi_{1}\left(S^{4} \backslash \iota\left(S^{2}\right)\right)=\mathbb{Z}$, then by [68] the complement $S^{4} \backslash \iota\left(S^{2}\right)$ has the homotopy type of $S^{1}$ and we obtain $B_{n}\left(S^{4} \backslash \iota\left(S^{2}\right)\right)=\mathbb{Z} \imath S_{n}$ and $\pi_{2}\left(\operatorname{Conf}_{n}\left(S^{4} \backslash \iota\left(S^{2}\right)\right)\right)=0$.

The fact that the braid groups for manifolds of dimension at least three have a simple structure as wreath products of the fundamental group of the manifold and the symmetric group means that, unlike the case of dimension two, there are no anyon states arising from representations of the braid groups of the ambient space. Indeed, for a system of $n$ identical particles on a smooth manifold $X$, with configuration space $\operatorname{Conf}_{n}(X)$, the set of irreducible unitary representations of the braid group $B_{n}(X)=\pi_{1}\left(\operatorname{Conf}_{n}(X)\right)$ labels inequivalent quantizations of the classical system. These can exhibit different possible statistics, which include bosons and fermions, as well as parastatistics, generalized parastatistics, and anyons. Parastatistics arise from higher dimensional representations of the symmetric groups, while fermions and bosons correspond to 1-dimensional representations. In the case of a simply connected manifold $X$ of dimension $\operatorname{dim} X \geq 3$, the braid groups are just symmetric groups by (3.5), hence one can only obtain fermions and bosons, or parastatistics. In particular, the only 1-dimensional (scalar) quantizations are either fermions or bosons. In cases of manifolds with $\operatorname{dim} X \geq 3$ with non-trivial fundamental groups, one obtains generalized parastatistics (see [47]). In the case of 2-dimensional manifolds, however, the situation is more interesting. On a 2-dimensional surface the braid group $B_{n}(X)$ is not simply a wreath product as in (3.5), but has a more interesting structure computed 
in $[21,22]$. This allows for more general exotic statistics, where even in the scalar case one can have statistics that are not fermions or bosons, but more general anyons, depending on an angle $\theta$. Non-abelian anyons arise from higher dimensional representations of the braid groups of 2-dimensional manifolds.

\subsubsection{Orbifold braid groups}

In the case of a 4-dimensional orbifold geometry $(M, \Sigma)$ with $M_{\text {reg }}=M \backslash \Sigma$ and 2dimensional $M_{\text {sing }}=\Sigma$, one can replace the braid groups $B_{n}\left(M_{\text {reg }}\right)=\pi_{1}\left(M_{\text {reg }}\right)$ 乙 $S_{n}$ with the orbifold braid group as in [77],

$$
B_{n}^{\text {orb }}(M)=\pi_{1}^{\text {orb }}(M) \curlywedge S_{n}=\pi_{1}^{\text {orb }}(M)^{n} \rtimes S_{n},
$$

with $\pi_{1}^{\text {orb }}(M)$ as in (3.1).

For example, in the case of the orbifold structure on $M_{\text {reg }}=S^{4} \backslash \mathbb{R} \mathbb{P}^{2}$ given by the Hitchin metrics of [45] with cone angle $2 \pi /(k-2)$, the orbifold braid groups are $B_{n}^{\text {orb }}(M)=S_{n}$ for $k$ even and $B_{n}^{\text {orb }}(M)=\mathbb{Z} / 2 \mathbb{Z} \imath S_{n}$ for $k$ odd, while for the AtiyahLeBrun orbifold structures on $M_{\text {reg }}=S^{4} \backslash S^{2}$ with cone angle $2 \pi / \nu$ the orbifold braid groups are $B_{n}^{\text {orb }}(M)=\mathbb{Z} / \nu \mathbb{Z} \imath S_{n}$.

Representations of orbifold braid groups of the 4-dimensional orbifold geometries $(M, \Sigma)$ can determine parastatistics and generalized parastatistics. Anyon representations associated to orbifold braid groups of 2-dimensional orbifolds were classified in [77], in terms of orbifold line bundles and Seifert invariants, in the context of quantum Hall models. Since anyons only arise from 2-dimensional geometries, the natural source of anyons in our models are the surfaces $\Sigma$ of orbifold points and the braid representations arising from associated surface braids. We explain this in the rest of this section.

\subsection{Surface braids}

Surface braids are a two-dimensional generalization of braids, initially introduced by Oleg Viro and developed by Kamada, $[54,55]$. A surface $m$-braid is a smooth 2-dimensional surface $S$, smoothly embedded in $D^{2} \times D^{2}$, such that the second projection $P_{2}: D^{2} \times D^{2} \rightarrow$ $D^{2}$ restricted to $S$ is an $m$-fold branched cover $P: S \rightarrow D^{2}$. The preimage $P_{2}^{-1}\left(\partial D^{2}\right) \cap S \subset$ $D^{2} \times S^{1}$ is a closed ordinary $m$-braid $\beta$. (Note: the terminology "surface braid" we use here is often used in the literature for the more restricted case where $\beta$ is the trivial braid, with "braided surface" used for this more general case, [55].)

Let $b(S) \subset D^{2}$ denote the set of branch points of the $m$-fold branched covering map $P: S \rightarrow D^{2}$. Let $\gamma(t)$ be a path in $D^{2} \backslash b(S)$ that represents a class in the fundamental group $\pi_{1}\left(D^{2} \backslash b(S)\right)$, computed, for example, with respect to a base point on the boundary $\partial D^{2}$. Taking

$$
\rho_{S}(\gamma)(t):=P_{1}\left(S \cap P_{2}^{-1}(\gamma(t))\right),
$$

where $P_{i}: D^{2} \times D^{2} \rightarrow D^{2}$ are the two projections, determines a path $\operatorname{in}_{\operatorname{Conf}}\left(D^{2}\right)$. This determines the braid representation

$$
\rho_{S}: \pi_{1}\left(D^{2} \backslash b(S)\right) \rightarrow \pi_{1}\left(\operatorname{Conf}_{m}\left(D^{2}\right)\right)=B_{m}\left(D^{2}\right) .
$$


A closed surface braid is similarly defined in $[54,55]$ as a smoothly embedded $S$ in $D^{2} \times S^{2}$, such that the restriction to $S$ of the projection to $S^{2}$ is an $m$-fold branched covering map $P: S \rightarrow S^{2}$. In our setting, we consider a more general form of closed surface braid, where $S^{2}$ is replaced by an arbitrary compact smooth 2-dimensional $\Sigma$. In particular, we consider the setting as above, where $\Sigma$ is a smoothly embedded smooth compact 2dimensional manifold in a compact 4-dimensional manifold $M$. If $\Sigma$ has several connected components, we focus on the neighborhood of only one component. Let $\mathcal{N}(\Sigma)$ be a tubular neighborhood of $\Sigma$ in $M$. Locally, over an open ball $D^{2} \subset \Sigma$, the tubular neighborhood is isomorphic to a product $D^{2} \times D^{2}$. By indentifying $\mathcal{N}(\Sigma)$ with the unit disc bundle of the normal bundle of the embedding $\Sigma \hookrightarrow M$, we write $P_{\mathcal{N}}: \mathcal{N}(\Sigma) \rightarrow \Sigma$ for the corresponding projection with fiber $D^{2}$. We define a closed surface braid in $M$ as an embedded surface $S$ in $\mathcal{N}(\Sigma)$ such that the restriction to $S$ of the projection $P_{\mathcal{N}}: \mathcal{N}(\Sigma) \rightarrow \Sigma$ is a $m$-fold branched cover $P: S \rightarrow \Sigma$. In the case where $\Sigma$ is an unknotted $S^{2}$ this recovers the original formulation of closed surface braids of Viro and Kamada.

Let $a_{1}, \ldots, a_{g}, b_{1}, \ldots, b_{g}$ be a set of generators for $\pi_{1}(\Sigma)$, where $g=g(\Sigma)$ is the genus. Consider a choice of representatives $a_{i}(t), b_{i}(t)$ given by paths in $\Sigma$. Let $P_{\mathcal{N}}: \mathcal{N}(\Sigma) \rightarrow \Sigma$ be the projection as above and let $S \subset \mathcal{N}(\Sigma)$ be a surface $m$-braid. Then $P_{\mathcal{N}}^{-1}\left(a_{i}\right) \cap S$ and $P_{\mathcal{N}}^{-1}\left(b_{i}\right) \cap S$ are closed ordinary $m$-braids $\beta_{a_{i}}, \beta_{b_{i}}$ in $D^{2} \times a_{i}=D^{2} \times S^{1}$ and $D^{2} \times b_{i}=D^{2} \times S^{1}$, respectively.

Given a closed surface $m$-braid $S$ in $\mathcal{N}(\Sigma)$, which is an $m$-fold branched cover of $\Sigma$ branched along a set of points $b(S) \subset \Sigma$, the associated braid representation is given by the group homomorphism

$$
\rho_{S}: \pi_{1}(\Sigma \backslash b(S)) \rightarrow \pi_{1}\left(\operatorname{Conf}_{m}\left(D^{2}\right)\right)=B_{m}\left(D^{2}\right),
$$

obtained as above by setting $\rho_{S}(\gamma)=P_{1}\left(S \cap P_{2}^{-1}(\gamma)\right)$, with $P_{2}$ the projection of the bundle $\mathcal{N}(\Sigma) \rightarrow \Sigma$ and $P_{1}$ the local projection in the fiber direction at a point in $S \cap P_{2}^{-1}(\gamma)$.

\subsection{Orbifold normal bundle}

In the case of the geometries we are considering, the embedded surface $\Sigma$ in the 4-manifold $M$ is the set of the orbifold points $M_{\text {sing }}=\Sigma$ of $M$. Thus, the normal bundle $\mathcal{N}(\Sigma)$ is in fact an orbifold bundle. We assume that $M$ is a good orbifold, covered by a compact smooth 4-dimensional manifold $X$ with an action of a finite group $G$, so that $M=X / G$. For simplicity, we can assume that the set of orbifold points is a connected surface and that $G=\mathbb{Z} / \nu \mathbb{Z}$. Then the orbifold bundle $\mathcal{N}(\Sigma)$ is orbifold covered by the normal bundle $\mathcal{N}(\tilde{\Sigma})$ of $\tilde{\Sigma}$, the preimage of $\Sigma$ in $X$. A section $\sigma$ of $\mathcal{N}(\Sigma)$ in general position intersects the zero section in a finite set of points $Q$. The preimage in $\mathcal{N}(\tilde{\Sigma})$ then determines a $\nu$-fold covering $S$ of $\Sigma$ branched at $Q$. We can identify $S$ with a surface braid, a $\nu$-fold cover of $\Sigma$ branched over $Q=b(S)$.

We obtain in this way, from an orbifold $(M, \Sigma)$ with cone angle $2 \pi \nu$, surface braids $S$ that are $\nu$-fold branched covers $P: S \rightarrow \Sigma$. In particular, if we have a fixed geometry $(M, \Sigma)$ that admits a family of orbifold edge-cone metrics with cone angles $2 \pi / \nu$ for any $\nu \in \mathbb{N}, \nu \geq 2$, we obtain surface $\nu$-braids $S$ for all $\nu \in \mathbb{N}, \nu \geq 2$, in the respective lifts 
$\mathcal{N}(\tilde{\Sigma})$ of the orbifold normal bundle $\mathcal{N}(\Sigma)$. Each of these surface braids determines a braid representation $\rho_{S}: \pi_{1}(\Sigma \backslash b(S)) \rightarrow \pi_{1}\left(\operatorname{Conf}_{\nu}\left(D^{2}\right)\right)=B_{\nu}\left(D^{2}\right)$.

In addition to considering sections of the orbifold normal bundle $\mathcal{N}(\Sigma)$, we can also consider multisections, given in local orbifold charts as $\mathbb{Z} / \nu \mathbb{Z}$-equivariant maps to $S^{n}(F)=F^{n} / S_{n}$, the symmetric product of the fiber $F \simeq D^{2}$ of the unit normal bundle $\mathcal{N}(\tilde{\Sigma})$ that orbifold covers $\mathcal{N}(\Sigma)$. Any such multisection $S$ determines an $\ell$-fold branched cover of $\tilde{\Sigma}$ branched where the multisection meets the diagonals in the symmetric product, hence an $\ell \nu$-fold branched cover of $\Sigma$, whose branch locus we again denote by $b(S)$. This gives an associated braid representation $\rho_{S}: \pi_{1}(\Sigma \backslash b(S)) \rightarrow \pi_{1}\left(\operatorname{Conf}_{\ell \nu}\left(D^{2}\right)\right)=B_{\ell \nu}\left(D^{2}\right)$. Thus, given an edge-cone metric with cone angle $2 \pi / \nu$, by considering all multisections of the orbifold normal bundle to $\Sigma$ that are in general position, we obtain braid representations in all the braid groups $B_{n}\left(D^{2}\right)$ with $n=\ell \nu$ for some $\ell \in \mathbb{N}$.

\subsection{Anyons and vortices}

In the description above, anyon states arise from surface braids given by multisections $S$ of the orbifold normal bundle of the surface $\Sigma$ of orbifold points in $M$. As such, these behave like extended objects. However, a localization to pointlike objects is taking place, through the fact that the associated braid representation depends on the branch points $b(S)$ and the structure of branched cover $S \rightarrow \Sigma$, which is described by local monodromy data.

Given the data of the surface with marked points $(\Sigma, b(S))$, one can consider associated vortex moduli spaces, in the form of symmetric products $\operatorname{Sym}^{n}(\Sigma, b(S))$, see [41] and section 5.7 of [79]. Note that the fundamental group of the symmetric products $\operatorname{Sym}^{n}(\Sigma, b(S))$ for $n>1$ are simply given by the abelianization of the fundamental group of the surface with marked points, see Remark 5.8 of [43] and Lemma 2.3 of [77]. However, the associated configuration spaces $\operatorname{Conf}_{n}(\Sigma \backslash b(S))$ determine braid groups $B_{n}(\Sigma, b(S)):=\pi_{1}\left(\operatorname{Conf}_{n}(\Sigma \backslash b(S))\right)$, with explicit Artin presentations as in [21, 22]. Thus, one can view the anyon states described above, arising from the braid representation $\rho_{S}: \pi_{1}(S \backslash b(S)) \rightarrow B_{\ell \nu}\left(D^{2}\right)$ as the first level of a more general construction that involves also braid representations where $\pi_{1}(S \backslash b(S))$ is replaced by the $B_{n}(\Sigma, b(S))$ for higher $n>1$. For the purpose of the present paper we focus only on the anyon states associated to the $n=1$ level and the braid representation $\rho_{S}: \pi_{1}(S \backslash b(S)) \rightarrow B_{\ell \nu}\left(D^{2}\right)$, which suffice, as we show in the next section, to obtain representations that are universal for quantum computing.

\section{Orbifold edge-cones as a quantum computer}

An important question regarding anyon systems is whether the associated braid representations are universal for quantum computing, which means unitary representations that span densely the group $\mathrm{SU}\left(2^{N}\right)$ of quantum gates for a system of $N$-qbits. In this section we address this question for the anyon systems constructed in the previous section and we show that, in one of the simplest cases of data $(M, \Sigma)$ given by the Atiyah-LeBrun orbifold edge-cone metrics on $S^{4} \backslash S^{2}$ with cone angle $2 \pi / \nu$ one does indeed obtain a braid representation that is universal for quantum computing. 
To put this question in context, note how intriguing connections between spacetime geometry and quantum computation have emerged recently in theoretical physics. The possibility of spacetime being emergent from quantum information and entanglement via quantum error correcting codes and tensor networks was proposed in the context of AdS/CFT correspondences, see [81]. The idea of the universe itself as a quantum computer was discussed in [72].

Here we take a different viewpoint and we suggest that the 4-dimensional orbifolds that arise as geometric models of matter allow for the presence of certain anyon representations that behave like a topological quantum computer, and we show that very simple examples can be constructed for which the resulting quantum computer is universal.

\subsection{Braided surfaces and universal quantum computers}

One of the main questions regarding physical systems that exhibit anyon statistics is whether they can determine unitary representations of the relevant braid groups that span densely the group $\mathrm{SU}\left(2^{N}\right)$, for a system of $N$-qbits. This property ensures that arbitrary quantum circuits can be approximated with a controllable error by elements in the representation, that is, that the representation determines a universal quantum computer, [59]. The Fibonacci anyons are an example of an anyon system satisfying this universality property, [90].

For a disc $D^{2}$, the braid group $B_{n}=B_{n}\left(D^{2}\right)$ is given by the Artin presentation

$$
\left.B_{n}=\left\langle\sigma_{1}, \ldots, \sigma_{n-1}\right| \sigma_{i} \sigma_{i+1} \sigma_{i}=\sigma_{i+1} \sigma_{i} \sigma_{i+1} \text { and } \sigma_{i} \sigma_{j}=\sigma_{j} \sigma_{i} \text { for }|i-j| \geq 2\right\rangle \text {. }
$$

A class of representations of the braid groups with very useful applications to quantum computation is given by the Jones representations of [52]. These are obtained by mapping, using the Kauffman bracket, the braid group algebra $\mathbb{F}\left[B_{n}\right]$ to the Temperley-Lieb algebra $T L_{n}(A)$, which is a quotient of the Hecke algebra $H_{n}(q)$, for $q=A^{-4}$. The algebra $T L_{n}(A)$ is then identified with a sum of matrix algebras $M_{n_{i}}(\mathbb{F})$, where $\mathbb{F}$ is the field of rational functions in the variable $A$, and restriction to these building blocks defines the Jones representations of the braid group, see the recent survey [26] for a quick overview. A construction in terms of braid groups of the Hecke algebra representations associated to the one-variable Jones polynomial was given in [64].

A crucial result in topological quantum computing is the fact that certain Jones unitary representations of the braid groups $B_{n}=B_{n}\left(D^{2}\right)$ determine a universal quantum computer, [37, 38].

In particular, the results of [37, 38], showing that Jones representations can give rise to a universal quantum computer, can be used to show that the Jones polynomial can be approximated efficiently by a quantum computer, see [26] and also [36, 86]. A new approach to the Jones polynomial, currently being developed in [7], presents a different possible approach to questions about its efficient computability, in the classical and quantum setting.

A property of quantum computation, which is very useful in order to obtain this type of density results, is the fact that the 1-qbit gates given by elements of $\mathrm{SU}(2)$ together with 
the CNOT gate

$$
\left(\begin{array}{llll}
1 & 0 & 0 & 0 \\
0 & 1 & 0 & 0 \\
0 & 0 & 0 & 1 \\
0 & 0 & 1 & 0
\end{array}\right)
$$

form a universal set for quantum computation, so that arbitrary gates in $\mathrm{SU}\left(2^{N}\right)$ can always be decomposed as tensor products of CNOTs and 1-qbit gates. Thus, in order to prove that certain unitary representations $\rho: B_{n} \rightarrow U(\mathcal{H})$ of braid groups are universal for quantum computing, it suffices to show that they approximate with arbitrary precision all the 1-qbit gates and CNOT.

For example, in [38] the state space $\left(\mathbb{C}^{2}\right)^{\otimes \ell}$ of $\ell$-qbits is embedded in the space $V\left(D^{2}, 3 \ell\right)$ assigned by a TQFT to the disc $D^{2}$ with $3 \ell$ marked points, so that the action of $B_{3 \ell}$ on $V\left(D^{2}, 3 \ell\right)$ is intertwined, via the embedding, with unitary operators acting on the state space $\left(\mathbb{C}^{2}\right)^{\otimes \ell}$. The TQFT considered in [38] is a Chern-Simons theory at a 5 -th root of unity. The action of $B_{3}$ on $V\left(D^{2}, 3\right)=\mathbb{C}^{2}$ gives the 1-qbit gates, while it is possible to obtain a desired 2-qbit gate by an approximation algorithm (Theorem 2.1 of [38]). This result is reformulated in [37] in terms of the Jones representations at $q=e^{ \pm 2 \pi i / 5}$. Note that in Chern-Simons theory level $\ell$ corresponds to $q^{2 \pi i /(\ell+2)},[92]$. This realization via Chern-Simons at a 5 -th root of unity of a braid group representation that is universal for quantum computing suffices for our purposes. It allows us to identify sufficiently simple examples of geometric models of matter $(M, \Sigma)$ with orbifold geometry, where the braid representations of the surface braids determined by multisections of the orbifold normal bundle to $\Sigma$ admit unitary representations that are universal for quantum computation.

Consider the example of the Atiyah-LeBrun orbifold edge-cone metric on $M_{\mathrm{reg}}=S^{4} \backslash S^{2}$ with cone angle $2 \pi / \nu$, as in [8]. The embedding of $\Sigma=S^{2}$ in $M=S^{4}$ is standard unknotted and the normal bundle $\mathcal{N}(\tilde{\Sigma})$ can be identified with $S^{2} \times D^{2}$ with $\mathbb{Z} / \nu \mathbb{Z}$ acting on the fiber $D^{2}$, and with $\mathcal{N}\left(S^{2}\right)$ the orbifold quotient. We use the notation $D_{f}^{2}$ and $D_{b}^{2}$ to distinguish between the 2-disc $D_{f}^{2}$ in the fiber of $\mathcal{N}\left(S^{2}\right)$ and the choice of a 2-disc $D_{b}^{2}$ in the base $S^{2}$. An $\ell$-multisection is then an $\ell \nu$-fold branched cover $S$ of $S^{2}$ in $S^{2} \times D_{f}^{2}$, that is, a closed surface $\ell \nu$-braid in the sense of $[54,55]$.

Given a closed surface $\ell \nu$-braid as above, choose one of the branch points $x_{0}$ in $S^{2}$ and a disc $D_{b}^{2} \subset S^{2}$ that is the complement of a small neighborhood of the chosen branch point. The restriction of the branch cover projection $S \rightarrow S^{2}$ of the closed surface braid to this disc determines a braided surface $\hat{S}$ in $D_{b}^{2} \times D_{f}^{2}$ that is an $\ell \nu$-fold branched cover of $D_{b}^{2}$ branched at $b(\hat{S})=b(S) \backslash\left\{x_{0}\right\}$. The intersection $\hat{S} \cap \partial D_{b}^{2} \times D_{f}^{2}$ is the closure of a braid $\beta \in B_{\ell \nu}$, which is in general nontrivial. Let $n=\# b(\hat{S})$ and let $\gamma_{1}, \ldots, \gamma_{n}$ be a set of generators (a Hurwitz arc system) of the fundamental group $\pi_{1}\left(D_{b}^{2} \backslash b(\hat{S})\right)$, computed with respect to a chosen basepoint on the boundary $\partial D_{b}^{2}$. The braid representation $\rho_{\hat{S}}$ : $\pi_{1}\left(D_{b}^{2} \backslash b(\hat{S})\right) \rightarrow B_{\ell \nu}\left(D_{f}^{2}\right)$ is then determined by the images $\beta_{k}=\rho_{\hat{S}}\left(\gamma_{k}\right)$, for $k=1, \ldots, n$. The element $\left(\beta_{1}, \ldots, \beta_{n}\right) \in B_{\ell \nu}\left(D^{2}\right)^{n}$ is the braid system of the braided surface $\hat{S}$.

For a braided surface $\hat{S}$ that is an $\ell \nu$-fold branched cover of $D^{2}$, one can give a characterization of all braid systems with the property that $\hat{S} \cap \partial D_{b}^{2} \times D_{f}^{2}$ is a given closed braid 
$\beta \in B_{\ell \nu}\left(D_{f}^{2}\right)$. Such braid systems are given by all the $n$-tuples $\left(\beta_{1}, \ldots, \beta_{n}\right) \in B_{\ell \nu}\left(D^{2}\right)^{n}$ with the property that each $\beta_{k}$ is a conjugate of a standard generator $\sigma_{i}$ of $B_{\ell \nu}\left(D^{2}\right)^{n}$ or an inverse $\sigma_{i}^{-1}$ and with $\beta=\beta_{1} \cdots \beta_{n} \in B_{\ell \nu}\left(D_{f}^{2}\right)$. The Hurwitz action of the braid group $B_{n}=B_{n}\left(D^{2}\right)$ on the $n$-fold product $B_{\ell \nu}\left(D^{2}\right)^{n}$ is given by

$$
\sigma_{i}:\left(\beta_{1}, \ldots, \beta_{i}, \beta_{i+1}, \ldots \beta_{n}\right) \mapsto\left(\beta_{1}, \ldots, \beta_{i-1}, \beta_{i} \beta_{i+1} \beta_{i}^{-1}, \beta_{i}, \beta_{i+2}, \ldots \beta_{n}\right) .
$$

Elements $\left(\beta_{1}, \ldots, \beta_{n}\right)$ that are in the same orbit of the Hurwitz action correspond to equivalent braided surfaces, that is, braided surfaces related by a fiber preserving diffeomorphism of $D_{b}^{2} \times D_{f}^{2}$ relative to the boundary $\partial D_{b}^{2} \times D_{f}^{2}$, see [55]. In particular, for $n=\ell \nu-1$, one can consider the standard braided surface of degree $\ell \nu$ with $\ell \nu-1$ branch points, which corresponds to the $n$-tuple of the standard generators $\left(\sigma_{1}, \ldots, \sigma_{n}\right) \in B_{n+1}^{n}$. There are in this case $(n+1)^{n-1}$ elements in the Hurwitz orbit, [46].

This example shows that, for each $\nu$ and $\ell$, there is a particular choice of a braided surface of degree $\ell \nu$ with $\ell \nu-1$ branch points whose braid system is the standard set of generators $\left(\sigma_{1}, \ldots, \sigma_{\ell \nu-1}\right)$ of the braid group $B_{\ell \nu}\left(D^{2}\right)$, which means that the braid representation for this braided surface recovers the full $B_{\ell \nu}\left(D^{2}\right)$. This fact, together with the existence of Jones unitary representations of the braid groups $B_{\ell \nu}\left(D^{2}\right)$ that span densely the groups $\mathrm{SU}\left(2^{N}\right)$ ensures that the braided surface configurations that arise in geometric models of matter given by Atiyah-LeBrun orbifold edge-cone metrics suffice to generate a universal quantum computer. Indeed, it suffices to take the orbifold edge-cone metric with cone angle $2 \pi / 3$ and apply the construction of [38] of unitary representations of $B_{3 \ell}$ based on the Chern-Simons TQFT at 5-th root of unity, which is universal for quantum computation.

One can then ask, for more general 4-dimensional orbifold geometries $(M, \Sigma)$, where $M$ has a self-dual Einstein orbifold edge-cone metric near $\Sigma$, whether it is always possible to find multisections $S$ of the orbifold normal bundle $\mathcal{N}(\Sigma)$, such that the image of the associated braid representation determines a universal quantum computer, or whether there are topological and geometric obstructions. In particular, one can look for 4-dimensional geometries related to the models of matter considered in $[3,10]$.

\subsection{Additional comments and questions}

The idea of a geometrization of the Skyrmion model originates in the work [11], where Skyrme field configurations in three dimensions with a given baryon number $k$ are generated via holonomies from SU(2) self-dual Yang-Mills instantons in four dimensions, with topological charge $k$. However, the model we discussed in the present paper is based on a different approach, developed more recently in $[6,10,12]$, where instead of considering Yang-Mills instantons in four dimensions, one considers gravitational instantons and certain more general classes of four-dimensional manifolds (like the algebraic surfaces considered in [10]) as the geometric models of Skyrmion-type hadronic physics. There are many significant differences between these approaches. For example, the relation between Skyrme fields and Yang-Mills instantons of [11] can be formulated in holographic terms that also provide a mechanism for the chiral symmetry breaking implemented at domain walls, [31]. In contrast, the geometric models of matter of $[6,10,12]$ in general do not 
admit a holographic description: this can be seen from the fact that these models include cases without boundary and with positive curvature (like the projective plane), contrary to the expected hyperbolicity and codimension one boundary of the holographic setting. It is still in principle possible that the specific models we focused on in this paper, which have an embedded surface of orbifold points, may be suitable for some form of holographic description: notice however that, unlike the usual holographic setting where the boundary has real codimension one with respect to the bulk, the setting we consider would require a more general form of holography based on a complex codimension one locus, such as a boundary divisor in the algebro-geometric sense, which is real codimension two in the bulk space. Investigating a possible approach to holography based on complex codimension one "boundary divisors" is beyond the scope of the present paper, though it represents an interesting problem in itself. In particular, the anyon states we focus on in this work arise from multisections of the orbifold normal bundle of the embedded surface of orbifold points. We can view the associated disk bundle as having locally two complex coordinates, one on the base Riemann surface and one in the fiber disks in the normal direction. A hypothetical holographic picture of the type mentioned above may regard the role of the complex coordinate in the fiber direction as analogous to a (complexified) RG scale, by analogy to the "holographic renormalization group" interpretation in AdS/CFT holography, [39]. A more in depth development of this topic would deserve a separate treatment, but we sketch here briefly an idea of a possible approach that will be expanded in mode detailed form elsewhere. These considerations should be regarded as speculative at this stage. The best approach to a possible boundary/bulk geometry appears to be the one based on tensor networks (see [81] and [32]), which carries with it a natural interpretation of the additional bulk coordinate as scale parameter with a multiscale entanglement renormalization ansatz (MERA), which is in general associated to geometric and topological properties like triangulations (see for instance [61] and [73]). In the setting we have introduced in this paper there is a natural geometric framework given by the multisections $S$ of the orbifold normal bundle $\mathcal{N}(\Sigma)$ of the embedded surface of orbifold points $\Sigma$ inside the 4-manifold $M$, together with their branched covering structure $S \rightarrow \Sigma$ branched along a finite set of points $b(S)$. A triangulation or more general decomposition of $\Sigma$ with vertices at $b(S)$ can be pulled back to consistent triangulations/decompositions on the multisection $S$, with weights corresponding to the data of the orbifold structure. To each such branched cover one would like to associate a tensor network and a MERA type diagram and RG flow picture that is consistent with the treatment of anyons in terms of tensor networks as in [82]. We can state this goal here as an open question and we hope to return to it in future work.

\section{Acknowledgments}

The first author received support from the Clay Mathematical Institute, Trinity College Cambridge, and the University of Edinburgh. The second author was partially supported by NSF grants DMS-1201512 and 1707882 and PHY-1205440 and the Perimeter Institute for Theoretical Physics. The second author would also like to thank Andrew Ranicki and Ida Thompson for their generous hospitality during her visits to the first author in Edinburgh. 
Open Access. This article is distributed under the terms of the Creative Commons Attribution License (CC-BY 4.0), which permits any use, distribution and reproduction in any medium, provided the original author(s) and source are credited.

\section{References}

[1] M.F. Atiyah, Configurations of points, in Topological methods in the physical sciences, London (2000), Roy. Soc. Lond. Phil. Trans. A 359 (2001) 1375.

[2] M.F. Atiyah, The non-existent complex 6-sphere, arXiv:1610.09366.

[3] M.F. Atiyah, Geometric Models of Helium, arXiv:1703.02532 [INSPIRE].

[4] M.F. Atiyah and J. Berndt, Projective planes, Severi varieties and spheres, in Surveys in differential geometry, vol. VIII, Int. Press (2003), Boston, MA (2002), pg. 1-27.

[5] M.F. Atiyah and R. Bielawski, Nahm's equations, configuration spaces and flag manifolds, Bull. Braz. Math. Soc. 33 (2002) 157 [math/0110112].

[6] M.F. Atiyah, G. Franchetti and B. Schroers, Time evolution in a geometric model of a particle, JHEP 02 (2015) 062 [arXiv:1412.5915] [INSPIRE].

[7] M.F. Atiyah and D. Jordan, A new approach to Jones-type invariants of knots, in preparation.

[8] M.F. Atiyah and C. LeBrun, Curvature, cones and characteristic numbers, Math. Proc. Cambr. Phil. Soc. 155 (2013) 13 [arXiv:1203.6389].

[9] M.F. Atiyah and N.J. Hitchin, The geometry and dynamics of magnetic monopoles, Princeton University Press (1988).

[10] M.F. Atiyah and N.S. Manton, Complex Geometry of Nuclei and Atoms, arXiv:1609.02816 [INSPIRE].

[11] M.F. Atiyah and N.S. Manton, Skyrmions From Instantons, Phys. Lett. B 222 (1989) 438 [INSPIRE].

[12] M. Atiyah, N.S. Manton and B.J. Schroers, Geometric Models of Matter, Proc. Roy. Soc. Lond. A 468 (2012) 1252 [arXiv:1108.5151] [INSPIRE].

[13] M.F. Atiyah and G. Segal, On equivariant Euler characteristic, J. Geom. Phys. 6 (1989) 671.

[14] M. Atiyah and P. Sutcliffe, The Geometry of point particles, Proc. Roy. Soc. Lond. A 458 (2002) 1089 [hep-th/0105179] [INSPIRE].

[15] M. Atiyah and P. Sutcliffe, Polyhedra in physics, chemistry and geometry, Milan J. Math. 71 (2003) 33 [math-ph/0303071] [INSPIRE].

[16] D. Auroux, Symplectic 4-manifolds as branched coverings of $\mathbb{C P}^{2}$, Invent. Math. 139 (2000) 551.

[17] I. Bakas, Anomalies, instantons and chiral symmetry breaking at a Lifshitz point, PoS (CORFU2011) 081 [arXiv: 1204.3833] [INSPIRE].

[18] I. Bakas and D. Lüst, Axial anomalies of Lifshitz fermions, Fortsch. Phys. 59 (2011) 937 [arXiv:1103.5693] [INSPIRE]. 
[19] J. Bellissard, Noncommutative geometry and quantum Hall effect, in Proceedings of the International Congress of Mathematicians, vol. 1-2, Zürich (1994), pg. 1238-1246, Birkhäuser, Basel (1995).

[20] M.V. Berry and J.M. Robbins, Indistinguishability for quantum particles: spin, statistics and the geometric phase, Proc. Roy. Soc. Lond. A 453 (1997) 1771.

[21] J. Birman, On braid groups, Commun. Pure Appl. Math. 22 (1969) 41.

[22] J. Birman, Mapping class groups and their relationship to braid groups, Commun. Pure Appl. Math. 22 (1969) 213.

[23] L.M. Brown ed., Feynman's thesis - A New Approach to Quantum Theory, World Scientific (2005).

[24] A.L. Carey, K.C. Hannabuss and V. Mathai, Quantum Hall effect and noncommutative geometry, J. Geom. Symmetry Phys. 6 (2006) 16.

[25] F. Dahia and C. Romero, The embedding of the space-time in five-dimensions: An Extension of Campbell-Magaard theorem, J. Math. Phys. 43 (2002) 5804 [gr-qc/0109076] [InSPIRE].

[26] C. Delaney, E.C. Rowell and Z. Wang, Local unitary representations of the braid group and their applications to quantum computing, arXiv:1604.06429.

[27] P. Deligne, Le groupe fondamental du complément d'une courbe plane n'ayant que des points doubles ordinaires est abélien (d'après W. Fulton), Bourbaki Seminar, vol. 1979/80, pp. 1-10, Lect. Notes Math., Vol. 842, Springer (1981).

[28] A. Dimca, Singularities and Topology of Hypersurfaces, Universitext, Springer (1992).

[29] S. Donaldson and R. Friedman, Connected sums of self-dual manifolds and deformations of singular spaces, Nonlinearity 2 (1989) 197.

[30] H.F. Dowker and R.S. Garcia, A handlebody calculus for topology change, Class. Quant. Grav. 15 (1998) 1859 [gr-qc/9711042] [InSPIRE].

[31] M. Eto, M. Nitta, K. Ohashi and D. Tong, Skyrmions from instantons inside domain walls, Phys. Rev. Lett. 95 (2005) 252003 [Erratum ibid. 95 (2005) 269904] [hep-th/0508130] [INSPIRE].

[32] G. Evenbly and G. Vidal, Tensor Network States and Geometry, J. Stat. Phys. 145 (2011) 891 [arXiv: 1106.1082].

[33] E. Fadell and L. Neuwirth, Configuration spaces, Math. Scand. 10 (1962) 111.

[34] S. Finashin, Knotting of algebraic curves in $\mathbb{C P}^{2}$, Topology 41 (2002) 47.

[35] G. Franchetti and N.S. Manton, Gravitational instantons as models for charged particle systems, JHEP 03 (2013) 072 [arXiv:1301.1624] [INSPIRE].

[36] M.H. Freedman, A. Kitaev and Z. Wang, Simulation of topological field theories by quantum computers, Commun. Math. Phys. 227 (2002) 587 [quant-ph/0001071] [inSPIRE].

[37] M.H. Freedman, M.J. Larsen and Z. Wang, The two-eigenvalue problem and density of Jones representation of braid groups, Commun. Math. Phys. 228 (2002) 177 [math/0103200].

[38] M.H. Freedman, M.J. Larsen and Z. Wang, A modular functor which is universal for quantum computation, Commun. Math. Phys. 227 (2002) 605.

[39] M. Fukuma, S. Matsuura and T. Sakai, Holographic renormalization group, Prog. Theor. Phys. 109 (2003) 489 [hep-th/0212314] [INSPIRE]. 
[40] W. Fulton, On the fundamental group of the complement of a node curve, Anna. Math. 111 (1980) 407.

[41] O. García-Prada, A direct existence proof for the vortex equations over a compact Riemann surface, Bull. London Math. Soc. 26 (1994) 88.

[42] G.W. Gibbons, H. Lü and C.N. Pope, Brane worlds in collision, Phys. Rev. Lett. 94 (2005) 131602 [hep-th/0501117] [INSPIRE].

[43] A. Grothendieck and M. Raynaud, Revêtements étales et groupe fondamental, in Séminaire de Géométrie Algébrique du Bois Marie, 1960-1961 (SGA I), Lect. Notes Math., vol. 224, Springer (1971).

[44] F. Hirzebruch and H. Höfer, On the Euler number of an orbifold, Math. Ann. 286 (1990) 255.

[45] N.J. Hitchin, A new family of Einstein metrics, in Manifolds and geometry, Pisa (1993), pg. 190-222, Sympos. Math., XXXVI, Cambridge University Press (1996).

[46] S.P. Humphries, Finite Hurwitz braid group actions for Artin groups, Isr. J. Math. 143 (2004) 189.

[47] T.D. Imbo, C.S. Imbo and E.C.G. Sudarshan, Identical Particles, Exotic Statistics and Braid Groups, Phys. Lett. B 234 (1990) 103 [InSPIRE].

[48] M. Iori and R. Piergallini, 4-manifolds as covers of the 4-sphere branched over non-singular surfaces, Geom. Topol. 6 (2002) 393 [math/0203087].

[49] L. Jacak, P. Sitko, K. Wieczorek and A. Wójs, Quantum Hall Systems: Braid groups, composite fermions and fractional charge, Oxford University Press (2003).

[50] J. Jacak, R. Gonczarek, L. Jacak and I. Jozwiak, Application of braid groups in $2 D$ Hall system physics: composite Fermion structure, World Scientific (2012).

[51] R. Jante and B.J. Schroers, Dirac operators on the Taub-NUT space, monopoles and SU(2) representations, JHEP 01 (2014) 114 [arXiv:1312.4879] [INSPIRE].

[52] V.F.R. Jones, Braid groups, Hecke algebras and type $I I_{1}$ factors, in Geometric methods in operator algebras, Kyoto (1983), vol. 123, pg. 242.

[53] S. Kallel and I. Saihi, Homotopy groups of diagonal complements, arXiv:1306.6272.

[54] S. Kamada, A characterization of groups of closed orientable surfaces in 4-space, Topology 33 (1994) 113.

[55] S. Kamada, Braid and Knot Theory in Dimension Four, American Mathematical Society (2002).

[56] T. Kawasaki, The index of elliptic operators over V-manifolds, Nagoya Math. J. 84 (1981) 135.

[57] H.J. Kim and D. Ruberman, Topological triviality of smoothly knotted surfaces in 4-manifolds, Trans. Am. Math. Soc. 360 (2008) 5869 [math/0610204].

[58] S. Kinoshita, On the Alexander polynomials of 2-spheres in a 4-sphere, Ann. Math. 74 (1961) 518.

[59] A. Kitaev, Quantum computations: algorithms and error correction, Russ. Math. Surv. 52 (1997) 1191.

[60] A. Kitaev, Anyons in an exactly solved model and beyond, Annals Phys. 321 (2006) 2 [INSPIRE]. 
[61] R. König, G. Kuperberg and B.W. Reichardt, Quantum computation with Turaev-Viro codes, Ann. Phys. 325 (2010) 2707 [arXiv: 1002. 2816].

[62] A. Kovalev and M. Singer, Gluing theorems for complete anti-self-dual spaces, Geom. Funct. Anal. 11 (2001) 1229 [math/0009158].

[63] V.S. Kulikov, A geometric realization of C-groups, Russian Acad. Sci. Izv. Math. 45 (1995) 197.

[64] R.J. Lawrence, Homology representations of braid groups, Ph.D. Thesis, University of Oxford (1989), http://www.ma.huji.ac.il/ ruthel/papers/thesis.html.

[65] C. LeBrun, Edges, orbifolds, and Seiberg-Witten theory, J. Math. Soc. Japan 67 (2015) 979. [arXiv:1305.1960].

[66] C. LeBrun and M. Singer, A Kummer-type construction of self-dual 4-manifolds, Math. Ann. 300 (1994) 165.

[67] A. Lerda, Anyons: quantum mechanics of particles with fractional statistics, Lect. Notes Phys., vol. 14, Springer (1992).

[68] J. Levine, Unknotting spheres in codimension two, Topology 4 (1965) 9.

[69] A. Libgober, On the homotopy type of the complement to plane algebraic curves, J. Reine Angew. Math. 367 (1986) 103.

[70] J.E. Lidsey, C. Romero, R.K. Tavakol and S. Rippl, On applications of Campbell's embedding theorem, Class. Quant. Grav. 14 (1997) 865 [gr-qc/9907040] [INSPIRE].

[71] M.T. Lock and J.A. Viaclovsky, An index theorem for anti-self-dual orbifold-cone metrics, Adv. Math. 248 (2013) 698 [arXiv:1209.3243].

[72] S. Lloyd, Universe as quantum computer. How decoherence leads to complexity, Complexity 3 (1997) 32 .

[73] Z.-X. Luo, E. Lake and Y.-S. Wu, The Structure of Fixed-Point Tensor Network States Characterizes Pattern of Long-Range Entanglement, Phys. Rev. B 96 (2017) 035101 [arXiv: 1611.01140] [INSPIRE].

[74] M. Marcolli and V. Mathai, Twisted index theory on good orbifolds. I. Noncommutative Bloch theory, Commun. Contemp. Math. 1 (1999) 553 [math/9911102].

[75] M. Marcolli and V. Mathai, Twisted higher index theory on good orbifolds, II: fractional quantum numbers, Commun. Math. Phys. 217 (2001) 55 [math/9911103] [INSPIRE].

[76] M. Marcolli and V. Mathai, Towards the fractional quantum Hall effect: a noncommutative geometry perspective, in Noncommutative Geometry and Number Theory, pg. 235-261, Aspects Math., E37, Vieweg(2006).

[77] M. Marcolli and K. Seipp, Twisted index theory on orbifold symmetric products and the fractional quantum Hall effect, Adv. Theor. Math. Phys. 21 (2017) 451 [arXiv:1502.01314] [INSPIRE].

[78] B.G. Moishezon, Stable branch curves and braid monodromies, in Algebraic geometry, Chicago, IL (1980), pg. 107-192, Lect. Notes Math., vol. 862, Springer (1981).

[79] T. Mrowka, P. Ozsváth and B. Yu, Seiberg-Witten monopoles on Seifert fibered spaces, Commun. Anal. Geom. 5 (1997) 685 [math/9612221]. 
[80] E. Newman, L. Tamubrino and T. Unti, Empty space generalization of the Schwarzschild metric, J. Math. Phys. 4 (1963) 915 [inSPIRE].

[81] F. Pastawski, B. Yoshida, D. Harlow and J. Preskill, Holographic quantum error-correcting codes: Toy models for the bulk/boundary correspondence, JHEP 06 (2015) 149 [arXiv: 1503.06237] [INSPIRE].

[82] R.N.C. Pfeifer, P. Corboz, O. Buerschaper, M. Aguado, M. Troyer and G. Vidal, Simulation of anyons with tensor network algorithms, Phys. Rev. B 82 (2010) 115126 [arXiv: 1006.3532].

[83] C.N. Pope, The $\eta$ Invariant for Charged Spinors in Taub-NUT, J. Phys. A 14 (1981) L133 [INSPIRE].

[84] I. Satake, The Gauss-Bonnet theorem for V-manifolds, J. Math. Soc. Japan 9 (1957) 464.

[85] P. Scott, The geometries of 3-manifolds, Bull. London Math. Soc. 15 (1983) 401.

[86] P.W. Shor and S.P. Jordan, Estimating Jones polynomials is a complete problem for one clean qubit, Quant. Inform. Comput. 8 (2008) 681 [arXiv:0707.2831].

[87] R.D. Sorkin, Kaluza-Klein Monopole, Phys. Rev. Lett. 51 (1983) 87 [InSPIRE].

[88] A.H. Taub, Empty space-times admitting a three parameter group of motions, Ann. Math. 53 (1951) 472 [INSPIRE].

[89] W. Thurston, The Geometry and Topology of 3-manifolds, unpublished manuscript, available at http://library.msri.org/books/gt3m.

[90] S. Trebst, M. Troyer, Z. Wang and A. Ludwig, A short introduction to Fibonacci anyon models, Prog. Theor. Phys. Suppl. 176 (2008) 384 [arXiv:0902.3275].

[91] Z. Wang, Topological Quantum Computation, American Mathematical Society (2010).

[92] E. Witten, Quantum Field Theory and the Jones Polynomial, Commun. Math. Phys. 121 (1989) 351 [INSPIRE].

[93] O. Zariski, On the problem of existence of algebraic functions of two variables possessing a given branch curve, Am. J. Math. 51 (1929) 305.

[94] O. Zariski, Algebraic Surfaces, reprint of the second edition (1971), Classics in Mathematics, Springer (1995). 